\title{
miRNA deregulation targets specific pathways in leiomyosarcoma development: an in silico analysis
}

\author{
Clara Benna ${ }^{1,2^{*}}\left(\mathbb{D}\right.$, Senthilkumar Rajendran ${ }^{1}$, Marco Rastrelli ${ }^{3}$ and Simone Mocellin ${ }^{1,3}$
}

\begin{abstract}
Background: MicroRNA (miRNA) mediate post-transcriptional gene repression and are involved in a variety of human diseases, including cancer. Soft tissue sarcomas are rare malignancies with a variety of histological subtypes which may occur virtually anywhere in the human body. Leiomyosarcoma is one of the most common subtypes, shows a smooth muscle phenotype and its cancerogenesis is still unclear. The aim of our study was to investigate the potential role of miRNA differential expression in leiomyosarcoma development.
\end{abstract}

Methods: We first employed the Sarcoma microRNA Expression Database, a repository that describes the patterns of over 1000 miRNA expression in various human sarcoma types, to identify differentially expressed miRNA comparing leiomyosarcoma and smooth muscle samples. Subsequently, we identified putative target genes of those miRNAs with the TargetScan prediction tool. Finally, we evaluated whether the retrieved pool of putative targets was enriched in genes belonging to specific molecular pathways by means of the Enrichr analysis tool. Protein-protein network analysis was analyzed by means of the STRING web tool.

Results: Out of 1120 miRNAs tested, the expression of 301 miRNAs was statistically significantly different between leiomyosarcoma and smooth muscle samples. The hypothetical targets could be predicted for 172 miRNAs. 438 genes were predicted to be the targets with high confidence (cumulative weighted context score cut-off level less than -1.0) and analyzed for belonging to specific molecular pathways. Pathway analysis suggested that RNA Polymerase III, tRNA functions and synaptic neurotransmission (with special regard to dopamine mediated signaling) could be involved in leiomyosarcoma development.

Conclusions: Our results demonstrate that data mining of publicly available repositories can be useful to suggest molecular pathways underlying the pathogenesis of rare tumors such as leiomyosarcoma.

Keywords: Sarcoma, Leiomyosarcoma, microRNA, miRNA, Pathway analysis, Dopamine, RNA polymerase III, RNA pol III

\section{Background}

Leiomyosarcoma is the second most frequent type of soft tissue sarcoma (STS) accounting for approximately $14 \%$ of all cases and is defined as demonstrating a smooth muscle phenotype with immunohistochemistry $[1,2]$. This rare tumor may occur virtually anywhere in the human body, from the limbs and trunk to the viscera

\footnotetext{
*Correspondence: clara.benna@unipd.it

${ }^{1}$ Department of Surgery Oncology and Gastroenterology, University of Padova, Padua, Italy

Full list of author information is available at the end of the article
}

and the retroperitoneum. Surgery remains the mainstay of treatment of localized leiomyosarcoma, but disease recurrence occurs frequently and chemotherapy is poorly effective to control metastatic disease [3]. Therefore, new therapies are eagerly needed in order to improve the prognosis of these patients. To this aim, understanding the cascade of molecular events leading to the development and progression of leiomyosarcoma plays a pivotal role in the identification of therapeutic targets.

miRNAs are 22-25 nucleotide RNAs that mediate post-transcriptional gene repression [4]. They are 
involved in a variety of human diseases, including malignancies, such as endometrial cancer [5], colorectal cancer [6], testicular cancer [7], acute myeloid leukemia [8], lung carcinoma [9], lymphoma [10], breast cancer [11] and osteosarcoma [12]. They can exert oncogenic functions as overexpressed miRNAs or serve as tumor suppressors and are consequently downregulated in the respective malignancy [13-15].

In this regard, recently, leiomyosarcoma has also been studied, moreover Authors' attention has focused on miRNA signatures for their emerging potential as diagnostic biomarkers and for aiding subclassification [1618], nevertheless the knowledge on this field of research is still poor.

The aim of our study is to further dissect molecular pathways involved in leiomyosarcoma development and progression. In a previous analysis [19] we explored the possible relation between the circadian clock pathway and soft tissue sarcoma (with special regard to leiomyosarcoma) susceptibility in terms of genetic variability. Here, we adopted a different strategy. First, we employed Sarcoma microRNA Expression Database (S-MED) https://www.oncomir.umn. edu/SMED/basic_search.php, a repository that describes the patterns of over 1000 miRNAs expression in various human sarcoma types, to individuate differentially expressed miRNA comparing leiomyosarcoma and smooth muscle tissues [20]. Subsequently, we identified putative target genes of those miRNAs with TargetScan prediction tool http://www.targetscan .org/ [21]. Finally, we evaluated whether the retrieved pool of putative targets was enriched in genes belonging to specific pathways http://amp.pharm.mssm.edu/ Enrichr/ [22]. Finally, further insights in the interactions across gene products were evaluated by means of network analysis using the STRING web tool.

\section{Methods}

\section{Study design}

The objective of this study is to identify gene pathways whose expression is preferentially altered in leiomyosarcoma development. To achieve this goal we proceeded with the following 3 steps:

1. miRNAs finding: identification of differentially expressed miRNAs in leiomyosarcoma and smooth muscle tissues.

2. miRNAs to genes: identification of differentially expressed miRNAs target genes.

3. From genes to pathways: identification of gene pathways of differentially expressed miRNAs targets.

\section{1. miRNAs finding}

Sarcoma-microRNA Expression Database (S-MED) [20] was employed to retrieve miRNA expression data in leiomyosarcoma and in smooth muscle. S-MED is a repository that describes the patterns of miRNA expression found in various human sarcoma tumor types and select normal tissues. S-MED provides both Basic and Advanced data search options for exploration of the data by means of heat-maps and text formats. Raw data were extracted for each of the considered miRNA. miRNAs in which the expression was not available in both leiomyosarcoma and smooth muscle were excluded. For each miRNA Student's T-test was performed to assess significantly differentially expressed miRNAs between leiomyosarcoma and smooth muscle tissues. Bonferroni correction for multiple testing was applied to define the level of significance. P-values smaller than 4.50E-05 were considered significant.

\section{2. miRNAs to genes}

TargetScan (v7.0; targetscan.org) [21] on line tool was employed for predicting effective microRNA target sites in human mRNAs. TargetScan predicts biological targets of miRNAs by searching for the presence of conserved $8 \mathrm{mer}, 7 \mathrm{mer}$, and $6 \mathrm{mer}$ sites that match the seed region of each miRNA [23]. In mammals, predictions are ranked based on the predicted efficacy of targeting as calculated using cumulative weighted context ++ scores of the sites [21]. The context ++ score (CS) for a specific site is the sum of the contribution of 14 features [21]: site type, supplementary pairing, local AU, minimum distance, sRNA1A, sRNA1C, sRNA1G, sRNA8A, sRNA8C, sRNA8G, site8A, site8C, site8G, $3^{\prime}$ UTR length, SA, ORF length, ORF 8mer count, $3^{\prime}$ UTR offset 6mer count, TA (target site abundance), SPS (seed-pairing stability), $\mathrm{P}_{\mathrm{CT}}$ (probability of conserved targeting). The cumulative weighted CS cut-off was set up at -1.0 in order to minimize false positive associations between miRNA and their targets, as well as to yield a manageable number of targets to be considered with both enrichment and network analysis [24].

\section{From genes to pathways}

Once target genes were identified, we used them to perform pathway analysis in order to identify biological functions whose genetic perturbations can predispose to leiomyosarcoma development.

For pathway analysis purposes, we utilized gene set enrichment analysis (GSEA) as performed by the EnrichR web server [22]. Hypergeometric distribution was used to calculate the statistical significance of gene 
overlapping [25], followed by correction for multiple hypotheses testing (using the false discovery rate [FDR] method) [26]. Only pathways with a FDR $<0.05$ were considered of interest.

In contrast to pathways, networks are not based on specific biological functions but are built based on both direct (physical) and indirect (genetic) interactions between gene products (proteins). For network analysis, we utilized the STRING 11.0 web server [27]. The resulting network provides information of the degree of overall connectivity across imputed gene products (as quantified by the ratio between observed and expected interactions [a.k.a. "edges"] between proteins [a.k.a. "nodes"], and formally tested by means of a PPI enrichment test). Moreover, it suggests cluster of interacting proteins, which can help identify specific cell pathways.

\section{Results}

\section{Differentially expressed miRNAs}

The results are summarized in the flow chart in Fig. 1. In the first step of this study, we compared the expression between leiomyosarcoma and smooth muscle tissues of 1120 miRNAs whose expression data were stored in Sarcoma-microRNA Expression Database (S-MED). For each miRNA data were available for 32 samples (22 leiomyosarcoma tissues and 10 smooth muscle tissues). The expression of 301 miRNAs was statistically significantly different $(P-$ value $<4.5 \mathrm{E}-05)$ between neoplastic and normal tissues (see Additional file 1: Table S1). The hypothetical targets could be predicted for 172 miRNAs by the target prediction tool TargetScan. For 129 miRNAs, mostly star miRNAs (the complementary strand of the unstarred), target prediction was not possible: for this reason, those miRNAs were excluded from further analysis. Employing a cumulative weighted context score cut-off level of -1.0 the retrieved putative gene targets were 608 (see Additional file 2: Table S2). After removing duplicates (which is because two or more miRNAs can share one or more target genes), the predicted targets resulted 438 , which were considered for pathway analysis (see below section).

\section{From genes to pathways}

Primary gene set enrichment analysis suggested that the 438 putative miRNA targets are enriched in genes involved in RNA polymerase III and tRNA functions (as transcription initiation, elongation, termination and tRNA modification) and in synaptic neurotransmission. Interrogating the KEGG and Reactome databases, the EnrichR webtool returned the results reported in Table 1. Network analysis of proteins encoded by

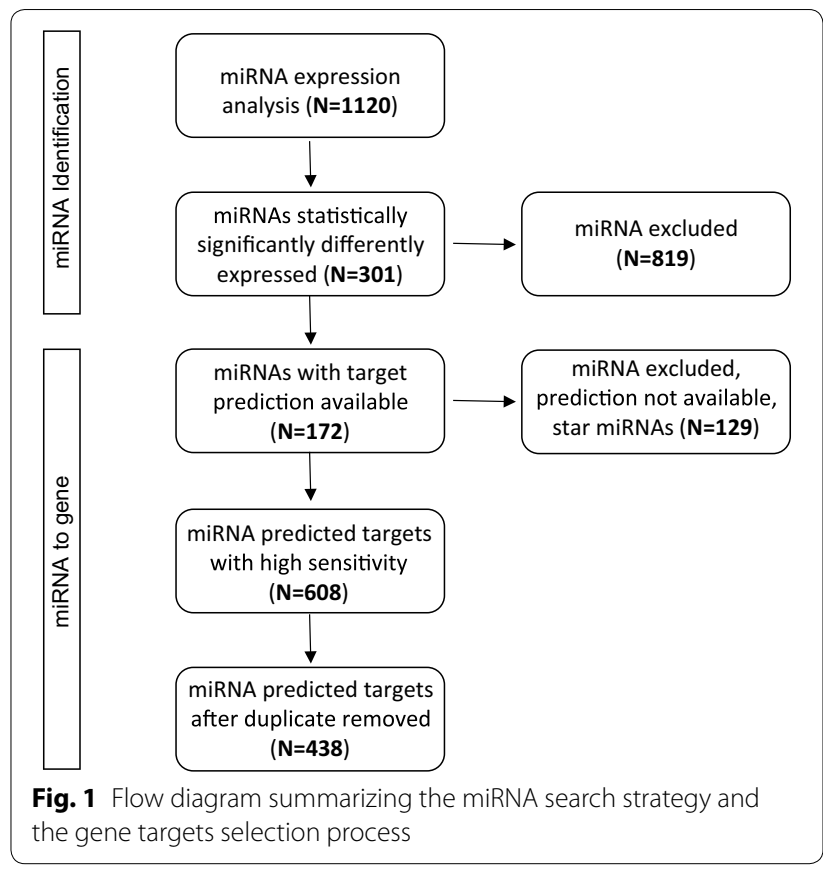

miRNA targets showed that the overall connectivity was significantly greater than expected (144/122 edges, $\mathrm{P}=0.03$ ) and confirmed the results obtained by enrichment analysis, especially underscoring the high number of interactions between proteins involved in neurotransmission (see Fig. 2).

\section{Discussion}

The aim of our study was to identify molecular pathways whose expression is particularly affected during leiomyosarcoma development. Our approach was first to identify miRNA which are statistically significantly down or up-regulated in leiomyosarcoma compared to smooth muscle, second to predict putative targets of those miRNA, and third to analyze if those putative target genes belonged to specific molecular pathways. Our results suggest that RNA Polymerase III, tRNA functions and synaptic neurotransmission could be altered during leiomyosarcoma development.

\section{RNA polymerase III, tRNA and cancer}

RNA polymerase III (pol III) is the largest RNA polymerase with the greatest number of subunits. It synthesizes a range of essential products, including tRNA, 5S rRNA and 7SL RNA, which are required for protein synthesis and trafficking. Moreover, while RNA polymerase I (pol I) synthesizes three ribosome subunits as a single precursor transcript that is processed into the final mature products, pol III produces MPR RNA, necessary for the processing; for a review see 
Table 1 Pathway analysis main findings: gene set enrichment analysis based on 438 miRNA target genes

\begin{tabular}{|c|c|c|c|c|}
\hline Pathway & Overlap & FDR & Genes & Database \\
\hline \multicolumn{5}{|l|}{ RNA polymerase III functions } \\
\hline RNA polymerase III transcription termination & $4 / 23$ & 0.0014 & NFIC; POLR3G; POLR3H; POLR2K & REACTOME \\
\hline RNA polymerase III chain elongation & $3 / 18$ & 0.0067 & POLR3G; POLR3H; POLR2K & REACTOME \\
\hline tRNA modification in the nucleus and cytosol & $4 / 39$ & 0.0102 & URM1; ADAT1; LCMT2; OSGEP & REACTOME \\
\hline RNA polymerase III transcription & $4 / 41$ & 0.0121 & NFIC; POLR3G; POLR3H; POLR2K & REACTOME \\
\hline RNA polymerase III & $3 / 32$ & 0.0324 & POLR3G; POLR3H; POLR2K & KEGG \\
\hline \multicolumn{5}{|l|}{ Synaptic neurotransmission } \\
\hline Dopaminergic synapse & $9 / 129$ & 0.0022 & $\begin{array}{l}\text { KCNJ6; ATF6B; KCNJ9; PPP2R2D; CACNA1A; GRIN2B; } \\
\text { CREB5; GNG13; MAPK13 }\end{array}$ & KEGG \\
\hline $\begin{array}{l}\text { Synapse formation and maturation (LGI-ADAM interac- } \\
\text { tions) }\end{array}$ & $3 / 14$ & 0.0032 & CACNG8; ADAM22; CACNG2 & REACTOME \\
\hline $\begin{array}{l}\text { Depolarization of the presynaptic terminal triggers the } \\
\text { opening of calcium channels }\end{array}$ & $2 / 13$ & 0.0318 & CACNA1A; CACNG2 & REACTOME \\
\hline $\begin{array}{l}\text { Neurotransmitter receptor binding and downstream } \\
\text { transmission in the postsynaptic cell }\end{array}$ & $7 / 142$ & 0.0370 & CACNG8; KCNJ6; KCNJ9; GRIK3; CACNG2; GRIN2B; GNG13 & REACTOME \\
\hline Morphine addiction & $5 / 91$ & 0.0496 & KCNJ6; KCNJ9; CACNA1A; GABRG1; GNG13 & KEGG \\
\hline \multicolumn{5}{|l|}{ Other pathways } \\
\hline Extrinsic pathway of fibrin clot formation & $2 / 5$ & 0.0046 & F7; TFPI & REACTOME \\
\hline Chemokine receptors bind chemokines & $5 / 56$ & 0.0075 & CCL22; CCL7; ACKR2; CXCL2; CXCL16 & REACTOME \\
\hline $\begin{array}{l}\text { TP53 regulates transcription of genes involved in G1 cell } \\
\text { cycle arrest }\end{array}$ & $2 / 13$ & 0.0318 & CCNE1; ZNF385A & REACTOME \\
\hline $\begin{array}{l}\text { Regulation of TP53 activity through association with co- } \\
\text { factors }\end{array}$ & $2 / 14$ & 0.0366 & ZNF385A;TP73 & REACTOME \\
\hline Hematopoietic cell lineage & $5 / 88$ & 0.0440 & FCER2; CSF2; TFRC; ITGA1; CD3E & KEGG \\
\hline Protein digestion and absorption & $5 / 90$ & 0.0477 & COL1A1; SLC6A19; COL3A1; COL12A1; SLC8A2 & KEGG \\
\hline
\end{tabular}

FDR false discovery rate

$[28,29]$. Abnormal pol III activity has been proposed to be feature of cancer cells. rRNA and tRNA are overproduced consistently in different human cancers as ovarian [30] breast, lung and tongue carcinomas [31, 32]. Conversely, in healthy cells oncogenes and tumor suppressor signaling pathways, such as the PI3kinase/ TORC1, Ras/ERK, Myc, p53 and Rb pathways, regulate Pol III and tRNA synthesis. In particular, pol III transcription factor (TFIIIB) interacts with the tumor suppressors RB and p53 to limit pol III production [33, 34]. Regarding tRNA, a pathway not related to pol III has also been identified in our study, which is tRNA modification in the nucleus and cytosol. Wobble tRNA modifications are required during translation elongation and sustain proteome homeostasis. A recent work has highlighted the upregulation of the wobble uridine 34 (U34) tRNA cascade in cancer, which underlies the specific requirement for this pathway in tumor development [35]. It is plausible to suppose that a further mechanism of controlling pol III abundance and tRNA production and maturation is due to miRNAs. Our results support this hypothesis and suggest that evading this form of control could contribute to leiomyosarcoma development.

\section{Dopamine}

Dopamine is a monoamine neurotransmitter, synthesized from the amino acid tyrosine, which is transported from the liver to the brain via an active transport mechanism. Dopamine plays central role in pleasurable reward behavior, hormone secretion, sleep, mood, attention, learning, behavior, control of nausea and vomiting, and pain processing.

Due to extensive localization of dopamine receptor to brain areas and its role in wide range of functions, dopaminergic dysfunction has been implicated in the pathophysiology of mood disorders, schizophrenia, obsessive compulsive disorder, autism spectrum disorders, attention deficit-hyperactivity disorder, Tourette's syndrome, substance dependency, Parkinson's disease and other disorders [36, 37].

Dopamine receptors (DRs) belong to the family of seven transmembrane domain G-protein coupled receptors and are classified into D1-like (DR1) and D2-like receptor families (DR2) based on 


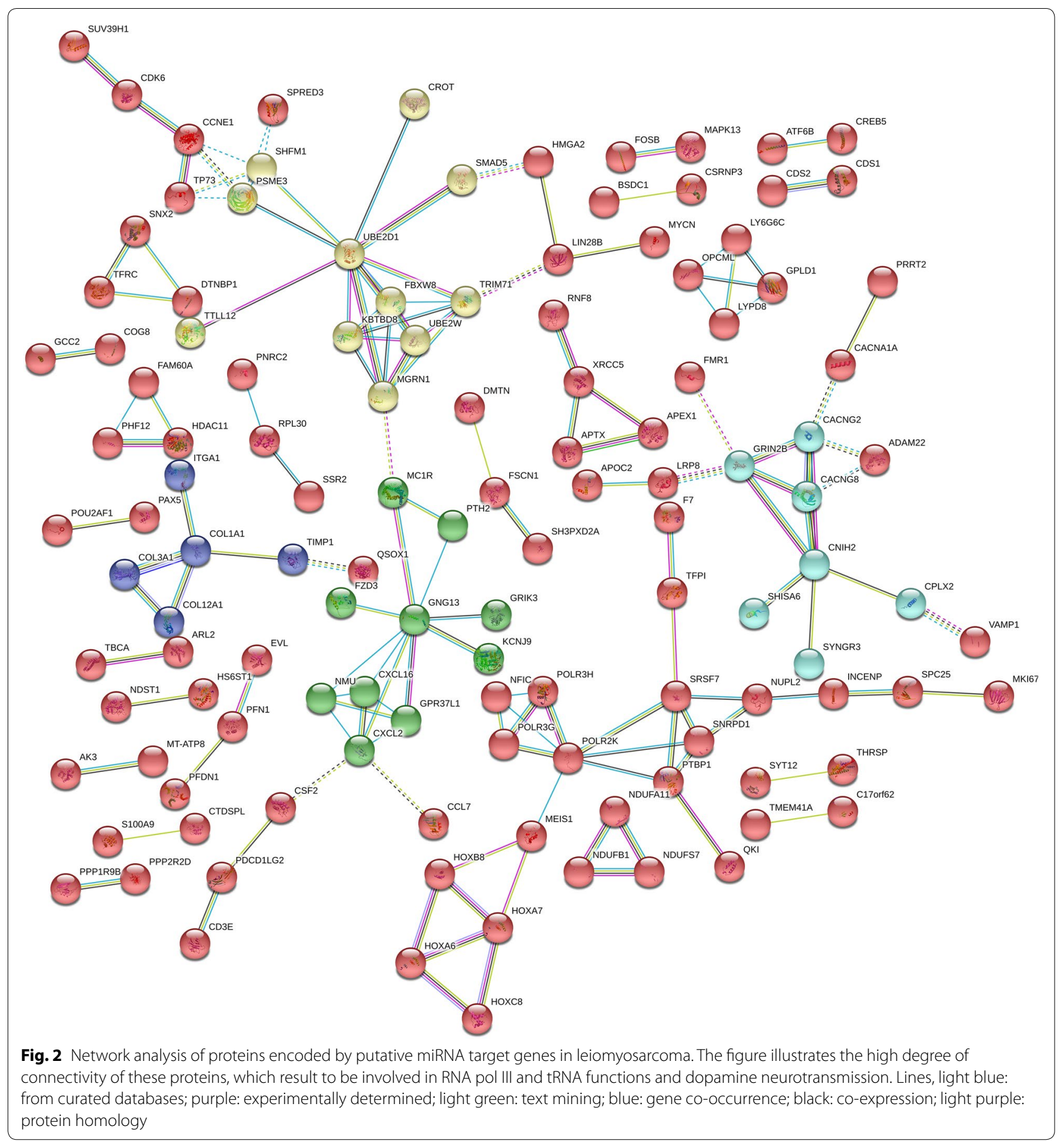

pharmacological properties, structure, and signal transduction system. DRs subtypes are expressed not only in brain areas, but also in many tissues and organs as kidney, heart, and the peripheral nervous. For this reason, this catecholamine also modulates cardiovascular function, vascular tone, renal function, and gastrointestinal motility.

\section{Dopamine and smooth muscle}

In recent works, many Authors found that the dopamine DR1 receptors are expressed on airway smooth muscle and regulate smooth muscle force via cAMP activation of PKA [38]. Moreover, it is reported that DR1 activation inhibited proliferation of the vascular smooth muscle cells [39]. 


\section{Dopamine and osteosarcoma}

Gao et al. [40] results suggest that DR1 are expressed in the osteosarcoma cells and inhibit the proliferation of osteosarcoma cells by the down-regulation of the ERK $1 / 2$ and PI3K-Akt pathways. In a different study, the same research group [41] suggested that activation of DR1 induces osteosarcoma cell apoptosis via changes to the MAPK pathway. The Authors proposed DR1 as a novel target for the treatment of osteosarcoma.

Here, the results of the present study support the hypothesis that dopamine pathway is involved in sarcoma growth and development and in particular in leiomyosarcoma.

\section{Potassium voltage-gated channel and cancer}

Considering each of the single target genes listed in this pathway, we found two potassium voltage-gated channel: Potassium Voltage-Gated Channel Subfamily J Member 6 (KCNJ6) and Potassium Voltage-Gated Channel Subfamily J Member 9 (KCNJ9). The ultimate effect of DR1 can be excitation (via opening of sodium channels) or inhibition (via opening of potassium channels). Voltagegated potassium channels (Kv), encoded by 40 genes in humans, are the largest subset of potassium channels gated by changes in the membrane potential [42]. Numerous studies have reported dysregulated potassium channel expression in human cancer [43]. In particular, few Authors focused on sarcoma: a study showed that that Kv1.3 voltage-gated potassium channels was upregulated in human osteosarcoma and downregulation of Kv1.3 suppressed osteosarcoma growth in vivo and osteosarcoma cell proliferation in vitro, accompanied by increased apoptosis [44]. Similar results were shown for the voltage-gated potassium channels Ether à go-go 1 and Kv1.5 [45, 46].

\section{Limitations}

This study provides useful insights for further studies, nevertheless it is limited by a number of weaknesses. The initial analysis was carried out on 1120 miRNAs for which data were available on SMED: nevertheless, identified human miRNA are at least twice as much (as reported by international databases such as MirBase, http://www.mirbase.org/), which leaves room for many more miRNAs to be evaluated in leiomyosarcoma pathogenesis. In addition, we could retrieve hypothetical target genes only for a miRNA subset because the TargetScan repository does not report data on star miRNAs, which, although usually degraded, sometimes also function as gene expression regulators $[47,48]$. Moreover, the analyses reported by the SMED web tool were based on 22 cases and 10 control samples: this small sample size does not protect against both false positive and false negative association results. As regards the target genes we have identified as potentially targeted by miRNA specifically deregulated in leiomyosarcoma, we could not validate our results in silico due to the lack of publicly available data on leiomyosarcoma. Consequently, dedicated experiments on human leiomyosarcoma samples are necessary to verify our hypothesis. Finally, though our results might suggest new therapeutic targets in the fight against leiomyosarcoma, experimental evidence proving the efficacy of this strategy is obviously warranted.

\section{Conclusions}

In soft tissue sarcomas, various miRNAs are differentially expressed, supporting the hypothesis that they could contribute to development, progression and invasion of this rare group of malignancies. Here, we focused on leiomyosarcoma, one of the most frequent types of soft tissue sarcomas. Overall our results suggest that there are pathways particularly targeted by miRNA altered expression which are already been linked to other malignancies, but are worth to be studied in leiomyosarcoma such as RNA pol III and tRNA functions and dopamine neurotransmission pathway. Expression and functional studies are needed to ascertain the role of these specific pathways in leiomyosarcoma development.

In general, our results demonstrate that data mining of publicly available repositories can be useful to suggest molecular pathways underlying the pathogenesis of rare tumors such as leiomyosarcoma.

\section{Additional files}

Additional file 1: Table S1. miRNAs retrieved in S-MED (Sarcoma-microRNA Expression Database), Student T-test P-val. Red: miRNAs with available targets prediction in TargetScan.

Additional file 2: Table S2. miRNAs targets predicted by TargetScan with cumulative weighted context score cut-off level of -1.0 or smaller.

\section{Abbreviations}

miRNA: microRNA; STS: soft tissue sarcoma; S-MED: Sarcoma microRNA Expression Database; CS: context++ score; GSEA: gene set enrichment analysis; FDR: false discovery rate; pol III: RNA polymerase III; pol I: RNA polymerase I;TFIII: pol III transcription factor; DRs: dopamine receptors; DR1: D1-like receptor family; DR2: D2-like receptor family.

\section{Acknowledgements}

Not applicable.

Authors' contributions

CB, SM: statistical analysis and manuscript writing; SR, MR: appraisal of manuscript. All authors read and approved the final manuscript.

\section{Funding}

University of Padova, BIRD168075, "Germline polymorphisms of candidate genes as predictor of risk and prognosis in patients with cutaneous melanoma and soft tissue sarcoma." 


\section{Availability of data and materials}

All data generated or analysed during this study are included in this published article and its Additional files.

\section{Ethics approval and consent to participate}

Not applicable.

\section{Consent for publication}

Not applicable.

\section{Competing interests}

The authors declare that they have no conflict of interests.

\section{Author details}

${ }^{1}$ Department of Surgery Oncology and Gastroenterology, University of Padova, Padua, Italy. ${ }^{2}$ Clinica Chirurgica I, Azienda Ospedaliera Padova, Padua, Italy. ${ }^{3}$ Surgical Oncology Unit, Istituto Oncologico Veneto (IOV-IRCCS), Padua, Italy.

Received: 14 March 2019 Accepted: 7 May 2019

Published online: 14 May 2019

\section{References}

1. Danielson LS, Menendez S, Attolini CS, Guijarro MV, Bisogna M, Wei J, et al. A differentiation-based microRNA signature identifies leiomyosarcoma as a mesenchymal stem cell-related malignancy. Am J Pathol. 2010:177(2):908-17

2. Stiller CA, Botta L, Brewster DH, Ho VKY, Frezza AM, Whelan J, et al. Survival of adults with cancers of bone or soft tissue in Europe-report from the EUROCARE-5 study. Cancer Epidemiol. 2018;56:146-53.

3. Ducimetiere F, Lurkin A, Ranchere-Vince D, Decouvelaere AV, Peoc'h M, Istier $L$, et al. Incidence of sarcoma histotypes and molecular subtypes in a prospective epidemiological study with central pathology review and molecular testing. PLOS ONE. 2011;6(8):e20294.

4. Mocellin S, Provenzano M. RNA interference: learning gene knock-down from cell physiology. J Transl Med. 2004;2(1):39.

5. Jurcevic S, Klinga-Levan K, Olsson B, Ejeskar K. Verification of microRNA expression in human endometrial adenocarcinoma. BMC Cancer. 2016;2(16):261.

6. Pichler M, Winter E, Ress AL, Bauernhofer T, Gerger A, Kiesslich T, et al. miR$181 \mathrm{a}$ is associated with poor clinical outcome in patients with colorectal cancer treated with EGFR inhibitor. J Clin Pathol. 2014:67(3):198-203.

7. Ling $H$, Krassnig L, Bullock MD, Pichler M. MicroRNAs in testicular cancer diagnosis and prognosis. Urol Clin North Am. 2016:43(1):127-34.

8. Gong JN, Yu J, Lin HS, Zhang XH, Yin XL, Xiao Z, et al. The role, mechanism and potentially therapeutic application of microRNA-29 family in acute myeloid leukemia. Cell Death Differ. 2014;21(1):100-12.

9. Yin Z, Xu M, Li P. miRNA-221 acts as an oncogenic role by directly targeting TIMP2 in non-small-cell lung carcinoma. Gene. 2017;15(620):46-53.

10. Troppan K, Wenzl K, Deutsch A, Ling H, Neumeister P, Pichler M. MicroRNAs in diffuse large B-cell lymphoma: implications for pathogenesis, diagnosis, prognosis and therapy. Anticancer Res. 2014;34(2):557-64.

11. Bertoli G, Cava C, Castiglioni I. The potential of miRNAs for diagnosis, treatment and monitoring of breast cancer. Scand J Clin Lab Invest Suppl. 2016:245:S34-9.

12. Zhao F, LV J, Gan H, Li Y, Wang R, Zhang H, et al. MiRNA profile of osteosarcoma with CD117 and stro-1 expression: miR-1247 functions as an oncomiRNA by targeting MAP3K9. Int J Clin Exp Pathol. 2015;8(2):1451-8.

13. Rupaimoole R, Slack FJ. MicroRNA therapeutics: towards a new era for the management of cancer and other diseases. Nat Rev Drug Discov. 2017;16(3):203-22.

14. Mocellin S, Pasquali S, Pilati P. Oncomirs: from tumor biology to molecularly targeted anticancer strategies. Mini Rev Med Chem. 2009;9(1):70-80.

15. Smolle MA, Leithner A, Posch F, Szkandera J, Liegl-Atzwanger B, Pichler M MicroRNAs in different histologies of soft tissue sarcoma: a comprehensive review. Int J Mol Sci. 2017;18(9):1960. https://doi.org/10.3390/ijms1 8091960

16. de Almeida BC, Garcia N, Maffazioli G, Gonzalez Dos Anjos L, Chada Baracat E, Candido Carvalho K. Oncomirs expression profiling in uterine leiomyosarcoma cells. Int J Mol Sci. 2017;19(1):52. https://doi. org/10.3390/ijms19010052.

17. Gonzalez Dos Anjos L, de Almeida BC, Gomes de Almeida T, Mourao Lavorato Rocha A, De Nardo Maffazioli G, Soares FA, et al. Could miRNA signatures be useful for predicting uterine sarcoma and carcinosarcoma prognosis and treatment. Cancers (Basel). 2018;10(9):315. https://doi. org/10.3390/cancers10090315.

18. Pazzaglia L, Novello C, Conti A, Pollino S, Picci P, Benassi MS. miR-152 down-regulation is associated with MET up-regulation in leiomyosarcoma and undifferentiated pleomorphic sarcoma. Cell Oncol (Dordr). 2017:40(1):77-88.

19. Benna C, Rajendran S, Spiro G, Tropea S, Del Fiore P, Rossi CR, et al. Associations of clock genes polymorphisms with soft tissue sarcoma susceptibility and prognosis. J Transl Med. 2018;16(1):338.

20. Sarver AL, Phalak R, Thayanithy V, Subramanian S. S-MED: sarcoma microRNA expression database. Lab Invest. 2010;90(5):753.

21. Agarwal V, Bell GW, Nam JW, Bartel DP. Predicting effective microRNA target sites in mammalian mRNAs. Elife. 2015. https://doi.org/10.7554/ elife.05005

22. Kuleshov MV, Jones MR, Rouillard AD, Fernandez NF, Duan Q, Wang Z, et al. Enrichr: a comprehensive gene set enrichment analysis web server 2016 update. Nucleic Acids Res. 2016;44(W1):W90-7.

23. Lewis BP, Burge CB, Bartel DP. Conserved seed pairing, often flanked by adenosines, indicates that thousands of human genes are microRNA targets. Cell. 2005;120(1):15-20.

24. Riffo-Campos AL, Riquelme I, Brebi-Mieville P. Tools for sequence-based miRNA target prediction: what to choose? Int J Mol Sci. 2016:17(12):1987. https://doi.org/10.3390/ijms17121987.

25. Rivals I, Personnaz L, Taing L, Potier MC. Enrichment or depletion of a GO category within a class of genes: which test? Bioinformatics. 2007:23(4):401-7.

26. Storey JD, Tibshirani R. Statistical significance for genomewide studies. Proc Natl Acad Sci USA. 2003;100(16):9440-5.

27. Szklarczyk D, Morris JH, Cook H, Kuhn M, Wyder S, Simonovic M, et al. The STRING database in 2017: quality-controlled protein-protein association networks, made broadly accessible. Nucleic Acids Res. 2017:45(D1):D362-8.

28. White RJ. RNA polymerases I and III, non-coding RNAs and cancer. Trends Genet. 2008;24(12):622-9.

29. White RJ. RNA polymerase III transcription and cancer. Oncogene. 2004;23(18):3208.

30. Winter AG, Sourvinos G, Allison SJ, Tosh K, Scott PH, Spandidos DA, et al. RNA polymerase III transcription factor TFIIIC2 is overexpressed in ovarian tumors. Proc Natl Acad Sci USA. 2000;97(23):12619-24.

31. Chen W, Heierhorst J, Brosius J, Tiedge H. Expression of neural BC1 RNA: induction in murine tumours. Eur J Cancer. 1997;33(2):288-92.

32. Chen W, Bocker W, Brosius J, Tiedge H. Expression of neural BC200 RNA in human tumours. J Pathol. 1997;183(3):345-51.

33. White RJ, Trouche D, Martin K, Jackson SP, Kouzarides T. Repression of RNA polymerase III transcription by the retinoblastoma protein. Nature. 1996:382(6586):88-90.

34. Crighton D, Woiwode A, Zhang C, Mandavia N, Morton JP, Warnock LJ, et al. p53 represses RNA polymerase III transcription by targeting TBP and inhibiting promoter occupancy by TFIIIB. EMBO J. 2003;22(11):2810-20.

35. Rapino F, Delaunay S, Zhou Z, Chariot A, Close P. tRNA modification: is cancer having a wobble? Trends Cancer. 2017;3(4):249-52.

36. Arias-Carrion O, Stamelou M, Murillo-Rodriguez E, Menendez-Gonzalez M, Poppel E. Dopaminergic reward system: a short integrative review. Int Arch Med. 2010;3:24.

37. Ayano G. Dopamine: receptors, functions, synthesis, pathways, locations and mental disorders: review of literatures. J Mental Disord Treat 2016;2(2):2-5.

38. Mizuta K, Zhang Y, Xu D, Mizuta F, D'Ovidio F, Masaki E, et al. The dopamine D1 receptor is expressed and facilitates relaxation in airway smooth muscle. Respir Res. 2013;14:89.

39. Zeng $C$, Han $Y$, Huang $H, Y u C$, Ren $H$, Shi W, et al. D1-like receptors inhibit insulin-induced vascular smooth muscle cell proliferation via down-regulation of insulin receptor expression. J Hypertens. 2009;27(5):1033-41.

40. Gao J, Zhang C, Gao F, Li H. The effect and mechanism of dopamine D1 receptors on the proliferation of osteosarcoma cells. Mol Cell Biochem. 2017:430(1-2):31-6. 
41. Gao J, Gao F. Dopamine D1 receptors induce apoptosis of osteosarcoma cells via changes of MAPK pathway. Clin Exp Pharmacol Physiol. 2017:44(11):1166-8.

42. Huang $X$, Jan LY. Targeting potassium channels in cancer. J Cell Biol. 2014;206(2):151-62.

43. Williams S, Bateman A, O'Kelly I. Altered expression of two-pore domain potassium (K2P) channels in cancer. PLoS ONE. 2013;8(10):e74589.

44. Wu J, Zhong D, Wu X, Sha M, Kang L, Ding Z. Voltage-gated potassium channel Kv1.3 is highly expressed in human osteosarcoma and promotes osteosarcoma growth. Int J Mol Sci. 2013;14(9):19245-56.

45. Wu J, Zhong D, Fu X, Liu Q, Kang L, Ding Z. Silencing of Ether Ã go-go 1 by shRNA inhibits osteosarcoma growth and cell cycle progression. Int J Mol Sci. 2014;15(4):5570-81.

46. Wu J, Chen Z, Liu Q, Zeng W, Wu X, Lin B. Silencing of Kv1.5 gene inhibits proliferation and induces apoptosis of osteosarcoma cells. Int J Mol Sci. 2015;16(11):26914-26.
47. Burroughs AM, Ando Y, de Hoon MJ, Tomaru Y, Suzuki H, Hayashizaki $Y$, et al. Deep-sequencing of human Argonaute-associated small RNAs provides insight into miRNA sorting and reveals Argonaute association with RNA fragments of diverse origin. RNA Biol. 2011;8(1):158-77.

48. Jazdzewski K, Liyanarachchi S, Swierniak M, Pachucki J, Ringel MD Jarzab B, et al. Polymorphic mature microRNAs from passenger strand of pre-miR-146a contribute to thyroid cancer. Proc Natl Acad Sci USA. 2009;106(5):1502-5.

\section{Publisher's Note}

Springer Nature remains neutral with regard to jurisdictional claims in published maps and institutional affiliations.
Ready to submit your research? Choose BMC and benefit from:

- fast, convenient online submission

- thorough peer review by experienced researchers in your field

- rapid publication on acceptance

- support for research data, including large and complex data types

- gold Open Access which fosters wider collaboration and increased citations

- maximum visibility for your research: over $100 \mathrm{M}$ website views per year

At BMC, research is always in progress.

Learn more biomedcentral.com/submissions 